\title{
2. RII analytical framework
}

\subsection{THE ELEPHANT IN THE ROOM}

An RII performance management framework could help to harmonise and optimise RII-related practices and policies. To implement such a framework, a university would need to have an 'RII process model' fed by reliable information to monitor activities and outcomes. University-wide RII implementation plans could generate a level of commitment needed to generate the economies of scale and scope with regard to RII pathways that could lead to a more active and successful pursuit of RII development options. Rather than being hampered by perceived organisational obstacles and/or potential risks for individual career development, a university could focus on opportunities the local environment may bring and develop joint aspirations (win/win objectives and shared advantages). The organisational responses, arrangements and strategies of universities, aimed at addressing expectations and pressures to become more locally oriented, will be varied. They depend in large part on opportunities with regards organisational configurations and capabilities, but also on funding structures as well as RII-oriented incentive structures and reward systems.

Most of the existing impact analytics and assessment methodologies tend to focus on knowledge exploitation activities and outputs, notably on the impact of academic research on business sector R\&D and technological innovation, or on academic entrepreneurship and university spin-off companies. However, the potential impact of universities is much broader. The steady supply of human resources from local universities can be a key contributor to regional innovation systems. Universities can also play a role in providing entrepreneurial skills and thus foster the development of new innovative ventures. Teaching and training curricula at universities, as well as their academic scientific research, are increasingly designed to create long-term socio-economic impacts and societal benefits. Unfortunately, many of the university's socio-economic impacts and those wider benefits defy easy detection, comprehensive coverage, or systematic measurement. This is not surprising considering how interconnected universities and regions are and given the fact that they are all an integral part of a larger dynamic social system with a dizzying number of actors and a myriad of intangible interactions. So, we have 
to face the major analytical challenge that is glaring at us, "the elephant in the room': how to identify, describe and assess RII activities?

Developing an RII analytical framework that can capture at least some features and processes of that complex system is a very challenging undertaking. According to Markiewicz and Patrick (2016, pp. 1-2) such a framework:

- is both a planning process and a written product designed to provide guidance to the conduct of assessment functions (monitoring and evaluation) over the life span of an initiative;

- includes an overarching plan and a step-by-step guide to its operationalisation and application over time;

- defines the parameters of routine monitoring and periodic assessment that will take place;

- shows how quantitative data and/or qualitative information are collected, aggregated and analysed on a regular basis to support assessment processes and outcomes that address policy goals.

Any useful framework will put the university's mission portfolio at centre stage and contextualise its RII potential and performance within its local or regional socio-economic circumstances. The acceptability and usefulness of the framework will significantly increase if it complies with general principles and requirements described in Box 2.1.

\section{BOX 2.1 GENERAL METHODOLOGICAL PRINCIPLES AND TECHNICAL REQUIREMENTS OF AN RII ANALYTICAL FRAMEWORK}

Ideally the analytical framework should:

- be functional for all intended users;

- offer added value compared to other sources of information;

- deploy transparent methods, qualitative information ('narratives') and/ or quantitative data ('numbers');

- provide information and data that are 'fact-based' (verifiable, empirical);

- adopt 'best practices' with regards to analytics, information gathering, measurement models, data definitions and data sharing, benchmarking and peer-learning; 
- include acceptable performance indicators ${ }^{1}$ - based on either qualitative information ('narratives') or quantitative data ('numbers');

- focus on innovation outcomes rather than simple quantity of engagement;

- incorporate general 'background' information as well as university-dependent and region-dependent 'foreground' specificities;

- offer opportunities to develop and implement customised information sources and indicators for specific needs of producers (universities) or users (stakeholders, funders, others); and

- have a well-designed governance structure that contributes to learning within the university in question as well as others.

But what defines the boundaries of the possible? Clearly, the availability of empirical information presents a formidable hurdle. Any RII analytical framework will be clearly handicapped by the lack of such information, or its questionable validity and relevance. The more sophisticated the framework, the scarcer the useful information and statistical data becomes. Systematic, comprehensive frameworks are indeed doomed to fail as diagnostic devices - if only because the highly-anticipated end product, the regional innovation impact, tends to be a shape-shifting and elusive outcome that may or may not emerge somewhere down the line. It could take years before any clearly identifiable impact materialises. By then it would be hard to establish the provenance of that particular effect or benefit, let alone attribute it to a specific source within a university. Sometimes, one can trace an impact back to a single 'make or break' event, such as the first publication about a scientific discovery or patent application of a breakthrough technology. In most cases, the timeline and causality are unclear and impact will often be generated by complex interplays of many sources and (hidden) determinants. Retrospective studies of RII trajectories may reveal that chance and randomness played a decisive role.

How to design an empirically feasible analytical framework of RII performance in the face of such uncertainty, ambiguity, and missing information? Analysts and policymakers will have to grapple with the inevitable trade-offs: between accounts of the 'now' and views of the 'possible future', objectivity and subjectivity, detailed accounts or panoramic overviews, between measurement and opinions. The graphical presentation of the RII model, presented in Figure 2.1, provides guidance. It shows that assessments of the 'RII delivery space', as presented in that graphical overview, is merely the tip of the iceberg

1 Indicators are indirect measures of phenomena or objects that cannot be measured directly. These proxy measures can be employed to assess RII model components or achievements that are of an abstract nature or comprised of attributes that are difficult to operationalise unambiguously. 


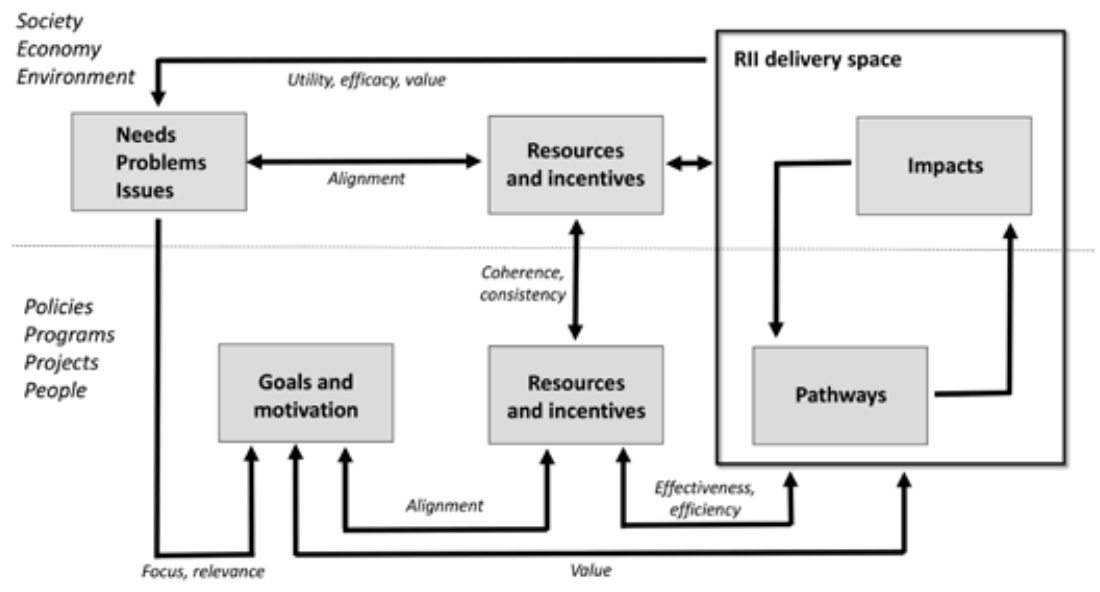

Source: Adapted from Jonkers et al. (2018); European Commission (2004).

\section{Figure 2.1 Analytical model for RII analytical framework}

and that the whole RII system will have to be reflected in the assessment in one way or another. The bottom section of this figure features general characteristics of RII system performance that can be monitored and assessed if the right kind of information is made available on its key components. The analytical model represents an 'RII value' oriented approach, emphasising discernible impacts on end users and gauging its value relative to the investments made and needs that are addressed. Operationalisation of the concept 'value' will vary; be it 'value for money', 'societal value' or something else, and depends on RII policy goals, organisational objectives or universities and many other factors.

How to assess the various dimensions of 'value' as well as other RII performance parameters such as 'efficiency' and 'relevance'? Relationships between RII supply and demand, the primary determiner of 'value' are often not straightforward, because the availability of new resources and knowledge can change the conditions of 'demand'. How to incorporate such dynamics? While collecting empirical evidence on impacts will largely rely on engagement narratives and showcasing, evidence on RII capacity seems much more amenable to measurement and quantitative performance indicators. In both cases, university-specific indicators and additional qualitative information will be needed to compile a sufficiently broad and in-depth RII profile of each university. Only then will the analytical framework be sufficiently developed to assess the interactions and connections between universities and their regions as well as their impact on innovation systems. 
Such an analysis or assessment can be 'formative' or 'summative'. Both can be part of organisational learning processes. The summative view is often backward-looking, focusing on past performance and achievements, and on whether and how goals or expectations have (not) been reached (see section 9.2 for further information on formative and summative approaches). The results feed into strategic decision-making or resource allocation. The formative approach tends to be forward-looking, focusing on possible opportunities or likely threats; outcomes of such assessments may serve as management input for strategic development or longer term organisational trajectories.

\subsection{GATHERING FACTS AND EVIDENCE: INFORMATION AND INDICATORS}

Availability and access to 'qualitative' information and 'quantitative' data sourced either from the university itself or from elsewhere - is of paramount importance for any workable RII analytical framework. Preferably it is easily accessible with meaningful, up-to-date and verifiable information. What kind of evidence-seeking indicators-based approach can be safely deployed for an up-to-date RII analytical framework? Is there any way of collecting or presenting information and data that is sufficiently reliable and robust? What kind of disclaimers need to be in place?

The origins of an RII may stretch back many years. Given that retrospective nature, RII analysis and assessments will necessarily involve historical information which may suffer from validity issues due to incomplete evidence, non-verifiable supporting documentation or selection biases. Retrospective narratives are geared towards the showcasing of success stories which allow us to recognise, with hindsight, RII successes and hopefully also identify the success factors that made the difference. Selective showcasing of 'regional impact stories' presents a format to convey key evidence framed within an organisational context and regional backdrop. There is a downside: information on obstacles, constraints or unfortunate circumstances that prevented RII from occurring are likely to be buried and lost for further analysis and organisational learning. Near-successes, let alone dismal failures, are often left out of the limelight. So, it is fair to assume that a significant share of RII-relevant information will be either missing, incomplete, outdated or non-verifiable. Although the use of narratives offers a qualitative sense, it also underscores the grave limitations of comparability, both across different cases within a university and across universities. Narrative-based 'qualitative' performance indicators are less suited for comparative purposes.

If accurate and valid information is out of reach for a full-blown RII assessment, we can search for proxies - the second-best option. Such 'indicators' do not always provide an accurate measure of an underlying phenomenon; hence 
due caution is required in analytical settings. Some indicators are probably misleading irrespective of their contexts, others could even be fundamentally flawed in particular circumstances. It is therefore important to be very clear about the limits of indicators: they will never cover all relevant dimensions of RII resources, pathways or performance.

Unsurprisingly, there are currently no agreed-upon indicators to gauge the RII potential of a university, let alone RII performance. 'Quantitative indicators', which are those relying exclusively on measurement and 'numbers', introduce a considerable risk of misrepresentation and underreporting. Even if measurements and quantitative indicators are only used as support tools to inform, enrich, or improve RII narratives we still need to be aware that numbers are a powerful messaging device in a narrative. Such performance measures impose a focus on those few components of RII capacity, impacts and spill-overs that are relatively easy to identify, categorise and measure. Relying on metrics tends to throw us back to crude, linear models because non-linear processes and flows are often beyond measurement. Numbers require context; it needs to be clear what they reflect or may signal.

\section{BOX 2.2 MULTIRANK INFORMATION PLATFORM}

\section{U-Multirank's 2019/2020 edition consists of seven 'Regional Engagement' performance indicators:}

- Income from regional sources;

- Student internships in the region;

- BA graduates working in region;

- MA graduates working in region;

- Graduates employed in the region;

- Strategic research partnerships in the region;

- Joint research publications with industrial partners located in the region.

The quantitative data for indicators 1 to 6 are based on self-appraisal reported information from each university; the numbers for indicator 7 derived from computations on information in international bibliographic databases.

The U-Multirank webpage ${ }^{2}$ contains more information on each of these indicators. $\mathrm{rs} /$.

See U-Multirank webpage: www.umultirank.org/about/methodology/indicato 
There are no readily available numbers on RII performance of individual universities. Fortunately, we are not entirely empty-handed as far as publicly accessible data is concerned with some bearing on RII. The online platform $U$-Multirank, one of the major ranking systems of universities worldwide, contains eight metrics-based, quantitative indicators that offer a baseline for measurement of RII-related performance (Box 2.2). The broad scope of these indicators, which cover education, research and employability, enables an evidence-based analysis of some aspects of a university's 'RII profile'.

U-Multirank's coverage of higher education institutions includes hundreds of universities in Europe or other higher education institutions. Not all registered universities are able to provide the requested information for each 'regional engagement' indicator. There is a substantial degree of missing data. As a result, the level of coverage across all U-Multirank registered universities tends to be 'moderate' in the case of the first six indicators. The last indicator provides 'complete' coverage, at least for those universities with a sufficiently large volume of research publication output. Each of these indicators can be useful to supplement an RII narrative, but also to provide an average performance level across universities. Take for example the share of 'BA graduates working in the region'. Selecting data from the U-Multirank's 2019 edition and those universities located within the EU27 (excluding the United Kingdom), this source provides data for 105 universities. Selecting the measurement year 2019, and surveying those who were students in 2015-2017, the share of BA graduates who found employment in the region was on average $64 \%$. The statistic for MA graduates (also for 112 universities) was 59\%.

Another example, 'Regional publications with industrial partners', comprises a much larger set of universities in the EU27. All these 590 organisations are 'research active', having produced at least 50 research publications in international scientific journals. The 'region' is clearly demarcated as a 50 $\mathrm{km}$ radius around the university's city of location. Any co-produced research publication with an R\&D partner from the business sector within this area is classified as regional. The average share of such regional co-publications, within all co-publications the university produced with the business sector, is $23 \%$. Many universities with strong R\&D ties to local industry tend to have shares of $30 \%$ or more (Tijssen, 2019).

U-Multirank indicators also invite direct comparisons across universities, but closer examination of definitions and operational parameters is required to assess the true relevance of these indicators for RII analysis, monitoring or assessment. Organisational comparisons and benchmarking can only be done with a reasonable degree of confidence if clear and convincing arguments exist as to why such an indicator reflects a feature that is sufficiently applicable or desirable in every university and region under investigation. 


\subsection{NARRATIVE WITH NUMBERS}

Shifting the analytical perspective, as much as possible, from past performance to the current situation solves some of the above-mentioned issues that may undermine the informational value of RII indicators. A focus on the current RII resources and pathways (such as supporting structures, processes, and communication channels) opens up possibilities to gauge the likelihood of future RIIs. Where narratives supply high-quality 'downstream' RII information, well-chosen indicators of RII pathway characteristics may be able to describe the 'upstream' potential to create such impacts but perhaps also possible opportunities or obstacles lurking behind the data. Moreover, RII capacity tend to be more amenable to measurement and aggregate-level comparability. There are several options for quantitative indicators, for example: graduate employment rate, sources of external income, R\&D cooperation contracts, students in entrepreneurship courses, number of university spin-off firms that have survived for five years, or resources spent on implementation of regional smart specialisation strategies. Selecting a suite of indicators, the resultant RII profile is likely to be representative of a university's general performance.

Synthesising these considerations and constraints boils down to three methodological principles that should drive 'smart' evidence-informed RII analysis and assessment:

- focus on up-to-date and reliable empirical evidence: collect qualitative and quantitative information on current RII resources and RII pathways (RII capacity) rather than on RII achievements;

- design and apply appropriate indicators: select only a limited number of broadly accepted proxy measures ('performance indicators') that reflect relevant attributes of the entire RII competence profile;

- contextualise the findings for meaningful interpretation and conclusions: assemble narratives and case studies that further describe and explain that RII competence profile, presenting regional background information but also organisational success factors and organisational obstacles.

Although metrics-based 'quantitative indicators' tend to carry a greater degree of objectivity and comparability, they come with various caveats in terms of validity, reliability and relevance. In view of the fact that several (potential) impacts can only be captured with qualitative information, rather than indicator-based statistical data, this three-pronged 'narrative with numbers' analytical framework presents a viable compromise between what we would like to have (in the ideal 'information on everything' world) and what is actually feasible given all the practical constraints we simply have to accept when studying a complex social system. This multi-method, multi-source approach 
has several advantages over purely qualitative case studies as they allow for a greater degree of objectivity, comparability and tracking of progress over time.

A narrative supported by indicators is a more appropriate analytical format to identify, categorise, interpret, and explain. To do so, the narratives must go beyond the numbers and should be more comprehensive. Ideally, narratives can be cross-checked and verified by independent sources. If numbers are used to help develop or substantiate narratives, their narrowness is not much of a problem; they illuminate some areas better than others. But even then, using such 'hard' performance indicators for RII policy purposes is always controversial, not only given their inherent limitations as proxies, but also because of the debatable nature of the associated policy goals. The inevitable tension between 'what is needed' and 'what is feasible' will have to be navigated. Regional innovation impacts can take years to materialise. In some very exceptional cases, they emerge quickly and are clearly visible, and the causal chain of events is easily established. These showcases can really bring home the message. However, in most other cases the 'signal' is weak and the causal relationships even weaker. The observed 'hard' empirical evidence captured in numbers might prove to be less robust or reliable than required, and thus the narrative is less convincing.

Neither 'quantitative' nor 'qualitative' are the perfect solution for an RII analytical framework, but a 'narrative with numbers' seems a satisfactory solution. However, this approach pushes some methodological boundaries into unchartered territory. It seems well-suited for purely descriptive purposes, and as an information filter 'heuristic', but could it also work as the empirical foundation of an RII analytical framework? Quantitative indicators need to be explicitly associated with RII characteristics of universities that are theoretically relevant, important for policy purposes, or both. Moreover, to turn an indicator into a performance measure is a very important decision from a management perspective and needs to be adequately supported. This is not a minor leap and would require a discussion of the performance criteria and associated characteristics that need to be promoted within universities, and how these elements map across the proposed performance indicators.

Even more challenging is the connection between RII assessment and funding decisions. It is not the same to develop a panel of indicators to provide a support for, say, aggregate analysis across universities or as a core component of a formula-based funding mechanism. The latter is especially problematic as it requires near-perfect alignment between RII policy objectives and the expected outcomes or impacts. As universities tend to 'chase the indicators' the effects could predictably gravitate towards 'what you measure is what you get'. Moreover, performance indicators for an RII funding formula should, in principle, be appropriate for comparative measurement. This means 
that the indicators are not influenced by the different contexts in which they are applied: regardless of context a higher value in an indicator would reflect, say, better performance. This is a tall order to achieve. Many of the traditional RII-related indicators, such as the number of spin-off companies produced by a university, respond to differences in the dynamics in regional business sectors or constraints imposed by national regulatory regimes.

Despite these pitfalls and caveats, our 'narrative with numbers' approach should prove applicable within and across the large variety of universities in Europe when guided by very cautious applications of its indicators, ensuring a robust mix of relevant information, and offering adequate opportunities for appropriate contextualisation. The framework's potential for real-life usage was tested in a series of case studies conducted in 2018 and 2019 among more than 20 research-active universities in Europe. As described in more detail in Part II, this pilot project offered valuable insights and lessons; not only to gauge the acceptability of the RII concept, but also on the feasibility of implementing that concept, and its four main analytical dimensions, in analytical settings and self-appraisal reporting by universities. All in all, the 'narrative with numbers' model seems useful for revealing the role of these universities in their regional innovation systems far beyond the information that numbers and rankings can offer. This reporting format allows organisations to both contextualise their performance - either within the context of a region's absorptive capacity and/ or the university's vision, mission, and strategy for its contributions to the region's socio-economic development. Either way, it is important to look at the 'bigger picture' as presented in the next chapter. 